Musées, Patrimoine et Culture scientifiques et techniques

$188 \mid 2020$

mars-avril 2020

\title{
Accompagner la rencontre entre Chercheurs et publics. L'expérience du Dôme
}

\section{Virginie Klauser}

\section{OpenEdition \\ Journals}

Édition électronique

URL : https://journals.openedition.org/ocim/3597

DOI : 10.4000/ocim.3597

ISSN : 2108-646X

Éditeur

OCIM

Édition imprimée

Date de publication : 1 mars 2020

Pagination : 8-13

ISSN : 0994-1908

Référence électronique

Virginie Klauser, « Accompagner la rencontre entre Chercheurs et publics. L'expérience du Dôme », La

Lettre de l'OCIM [En ligne], 188 | 2020, mis en ligne le 01 mars 2021, consulté le 17 juillet 2021. URL http://journals.openedition.org/ocim/3597 ; DOl : https://doi.org/10.4000/ocim.3597

Ce document a été généré automatiquement le 17 juillet 2021.

Tous droits réservés 


\section{Accompagner la rencontre entre Chercheurs et publics. L'expérience du Dôme}

Virginie Klauser

Opérationde médiationdu Dôme.

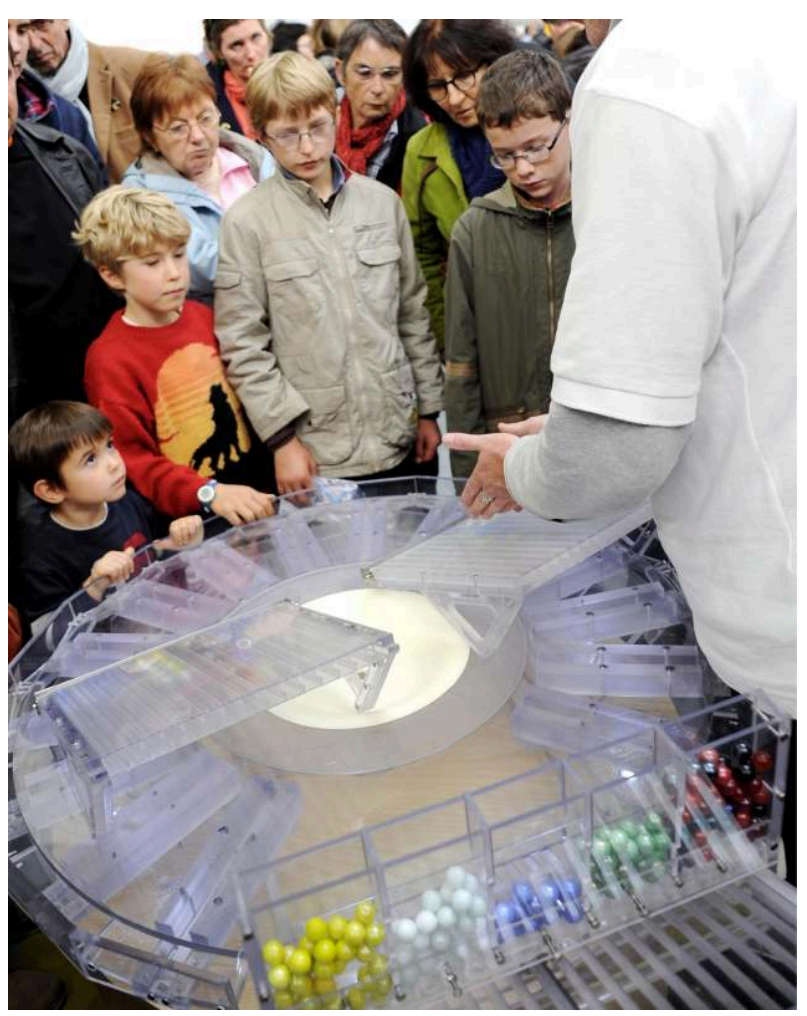

(c) Franck Castel 


\section{Se former en médiation scientifique pendant la thèse}

1 Que l'on soit biologiste, juriste, informaticien, linguiste, physicien ou philosophe, la culture scientifique et technique constitue une dimension fondamentale du travail de recherche. Présentation de thèse, entretien d'embauche, enseignement, recherche de fonds, médiatisation de la recherche et de ses résultats... Les opportunités de prise de parole du chercheur sont nombreuses mais communiquer ne s'improvise pas. Il faut savoir identifier le niveau de connaissances et les attentes de son public pour adapter sa présentation et la rendre dynamique.

2 Former la nouvelle génération de chercheurs, c'est former les doctorants et postdoctorants animés par une volonté de partager leur parcours avec un public néophyte. Pour les accompagner, le Dôme développe depuis 2006 un programme de formation à la médiation scientifique associant théorie et pratique de terrain avec le soutien de la Région Normandie. En 13 ans, plus de 500 doctorants sont passés par ces formations et ont relevé un défi: parler de façon compréhensible de l'actualité de la recherche normande devant un public non-expert.

Du côté du Dôme, les principaux objectifs, vis-à-vis de cette nouvelle génération, sont de donner les clés pour communiquer face à des publics différents dans des conditions variées et de mettre à profit les compétences acquises auprès des publics familiaux et scolaires. Du côté des doctorants, il s'agit d'utiliser ces compétences et ces expériences de mises en pratique sur le terrain, pour gagner en aisance et en capacité d'adaptation lors d'événements de médiation scientifique et de pouvoir également les réinvestir dans un cadre professionnel tels que l'enseignement ou les présentations orales.

4 L'offre doctorale proposant deux niveaux de formation, les jeunes chercheuses et chercheurs ont ainsi l'opportunité d'acquérir dans un premier temps les bases de la médiation scientifique puis d'approfondir leurs connaissances sur les outils innovants de présentation de leur thèse.

5 Ces formations théoriques se déroulent sur une journée durant laquelle les médiateurs du Dôme leur apportent des méthodes et des outils adaptés afin de dialoguer simplement, avec différents types de publics. Pour les doctorants désireux d'aller plus loin dans les formats de médiation, la formation «Se former aux outils innovants de présentation" bouscule la traditionnelle affiche pour leur apprendre à façonner un objet grâce aux machines à commande numérique du Fablab.

6 Lors de ces formations, les doctorants prennent généralement du recul sur leur manière de présenter leur sujet de thèse puisqu'ils découvrent de nouvelles approches, parfois avec des contraintes comme minuter un diaporama à la mode Pecha Kucha ${ }^{1}$ ou résumer sa thèse en quelques icônes. Ils prennent également conscience de l'importance de l'interactivité pour rendre une présentation plus dynamique et surtout conserver l'attention de l'auditoire.

7 C'est aussi l'occasion de faire des rencontres, de se confronter aux autres disciplines, de découvrir comment la démarche expérimentale est appliquée selon qu'on étudie l'astrophysique, la littérature, la psychologie, la chimie... Ces jeunes chercheurs se côtoient pour la première fois de façon conviviale, car l'ambiance y est détendue autour d'exercices ludiques basés souvent sur de la bande dessinée, sur des présentations décalées et sur des mises en situation interactives. Ils se rendent compte qu'ils partagent les mêmes valeurs, une envie de transmettre des connaissances, une passion 
commune pour la recherche, un besoin d'aller vers les autres pour partager leur expérience et surtout un désir d'inspirer ces collégiens ou lycéens qui cherchent leur voie en leur montrant que tout est possible lorsque l'on se donne les moyens. Enfin, ils se rejoignent sur un but commun: comment parler de ses recherches à un public néophyte ? C'est un premier pas vers la création d'une communauté doctorante que le Dôme souhaite installer en région.

Exemple d'outil de médiation, développé par Olivier Cortier lors d'une formation pour aborder l'étude des revêtements perméables pour la protection des sols et l'amélioration du cycle de l'eau en milieu urbain.

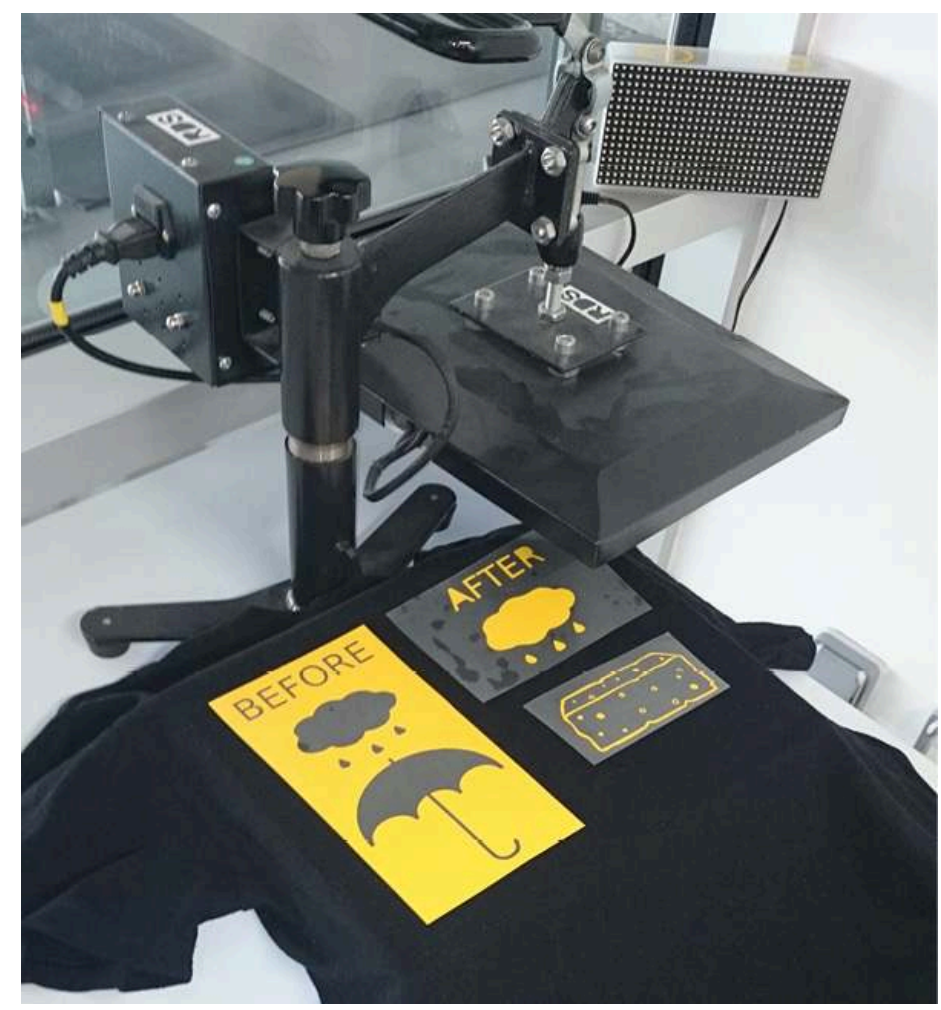

(c) Le Dôme/Virginie Klauser

Les open badges : outils de reconnaissance et de valorisation

Depuis 2017, le Dôme a rejoint le collectif Badgeons la Normandie - pionnier en France dans l'expérimentation des open badges - créés par les fondations Mozilla et Mac Arthur afin de combler un manque dans les outils de reconnaissance des apprentissages informels.

Véritable révolution dans le domaine de la reconnaissance des compétences et apprentissages informels, ils pourraient être mis en œuvre dans une grande variété de contextes et secteurs d'activités, par exemple pour bâtir des parcours de formation, trouver des emplois, rejoindre des communautés de pratique ${ }^{2}$, cartographier les compétences d'un territoire - en mettant en relation lieux, personnes et connaissances - ou rechercher de nouvelles opportunités d'apprentissage. 
Aujourd'hui, les badges numériques sont de plus en plus plébiscités et scrutés par les acteurs nationaux de l'éducation, de la formation ou de l'insertion sociale et professionnelle.

\section{Exemple d'application sur les activités de médiation des doctorants}

Pour le Dôme, les badges constituent un outil à la croisée de plusieurs activités, qu'il s'agisse de motiver et reconnaître la participation des publics, de la valoriser, de témoigner des apprentissages et découvertes dispensés au sein des activités. Fort de son positionnement à la croisée de la recherche, de la culture, de l'éducation formelle et informelle, le Dôme est en mesure d'affirmer une expertise et un rayonnement au-delà des frontières régionales.

Concrètement, l'open badge est une image numérique dans laquelle sont encodées des informations infalsifiables qui traduisent nos compétences, nos expériences, nos convictions, notre implication dans des projets, dans la société.

Un chantier a d'ailleurs été lancé pour récompenser la reconnaissance des contributions et des participations aux programmes menés avec la communauté scientifique, comme par exemple des badges pour reconnaître l'engagement et la formation acquise par des doctorants lors d'actions de culture scientifique. En 2019, des sessions d'ateliers et de temps de présentation ont été menés afin d'acculturer ce public aux open badges. L'objectif est de concevoir et prototyper les développements numériques pour l'usages des open badges avec et pour les doctorants.

Ainsi, deux badges « Formation » ont déjà été créés et testés avec ces derniers. La journée d'acquisition de connaissances est validée grâce à un niveau de badge « j'ai testé » et leur participation plus concrète dans la formation est reconnue par un niveau plus élevé «j'ai découvert ». Puis, fut créé un badge de terrain Atelier du chercheur « je peux faire » pour valider l'implication des chercheurs et chercheuses auprès des élèves (de la classe de $3^{\mathrm{e}}$ jusqu'en Terminale). Enfin, un badge Pint of science a été délivré afin de signifier l'engagement des scientifiques qui participent à l'opération sur Caen en diffusant la culture scientifique auprès d'un public néophyte - qui lui aussi a eu droit a son badge « j'ai pris une pinte avec des chercheurs ".

La réflexion se poursuivra au fur et à mesure des opérations et des retours avec les principaux intéressés.

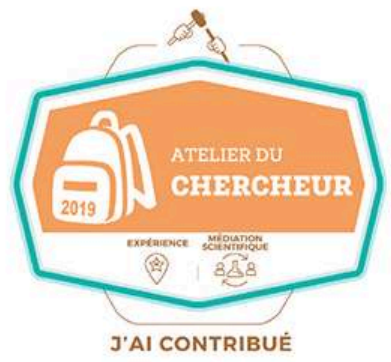

(C) Le Dôme
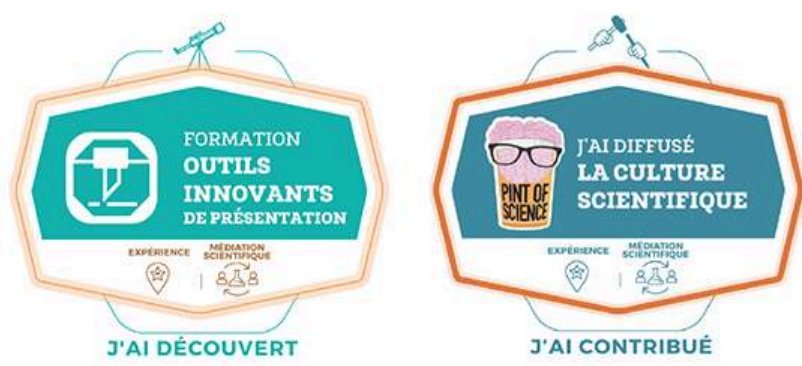


\section{Aller à la rencontre de son public} nouvelles compétences sur le terrain : savoir vulgariser sa thèse, extraire l'essentiel de ses travaux de recherche, être capable de mettre en vie son discours et interagir avec le public, savoir adapter son discours mais aussi prendre du plaisir en discutant avec un public profane.

9 Accompagnés par des médiateurs, les dispositifs de mise en situation sont divers afin de s'adresser à plusieurs typologies de public.

\section{Sensibiliser le public scolaire}

L'Atelier du chercheur est par exemple l'occasion pour de jeunes chercheurs de partir à la rencontre de collégiens et de lycéens pour parler métier, cursus et passion. Librement inspirée de l'Experimentarium de l'université de Bourgogne ${ }^{3}$, l'opération est née en 2006 à l'initiative de Cécile Malfray, alors médiatrice scientifique à Relais d'sciences. L'objectif est d'amener la recherche dans les territoires au plus près des publics et notamment des scolaires. En 13 années d'existence, ce sont plus de 13500 collégiens et lycéens de la région Normandie qui ont échangé en direct avec plus de 280 scientifiques.

11 Pour le Dôme, c'est l'opportunité de toucher un public scolaire éloigné géographiquement qui se déplace mal, même pour la Fête de la science, et d'encourager la dynamique de diffusion de la culture scientifique par les chercheurs. La motivation des doctorants repose sur le fait qu'ils pourront transmettre un message à la prochaine génération, profiter de cette occasion pour faire de la vulgarisation scientifique avec un jeune public néophyte et peut-être avoir l'occasion de se retrouver sur les bancs de leur ancienne école. C'est aussi l'opportunité pour des chercheurs d'horizons variés d'agir autour d'une manipulation ou d'un objet emblématique qui suscite le questionnement sur les représentations stéréotypées que les scolaires ont de la recherche et ses métiers. Qui sont les chercheurs? Quel est leur parcours? Comment travaillent-ils? Que cherchent-ils? À quoi ressemble leur quotidien? Sont-ils vraiment des hommes et des femmes comme les autres? 
Pour l'Atelier du chercheur, la doctorante Floriane Calocer a imaginé un jeu de cartes pour parler épidémiologie : les lycéens de la Ferté-Macé découvrent les maladies en cherchant les symptômes associés.

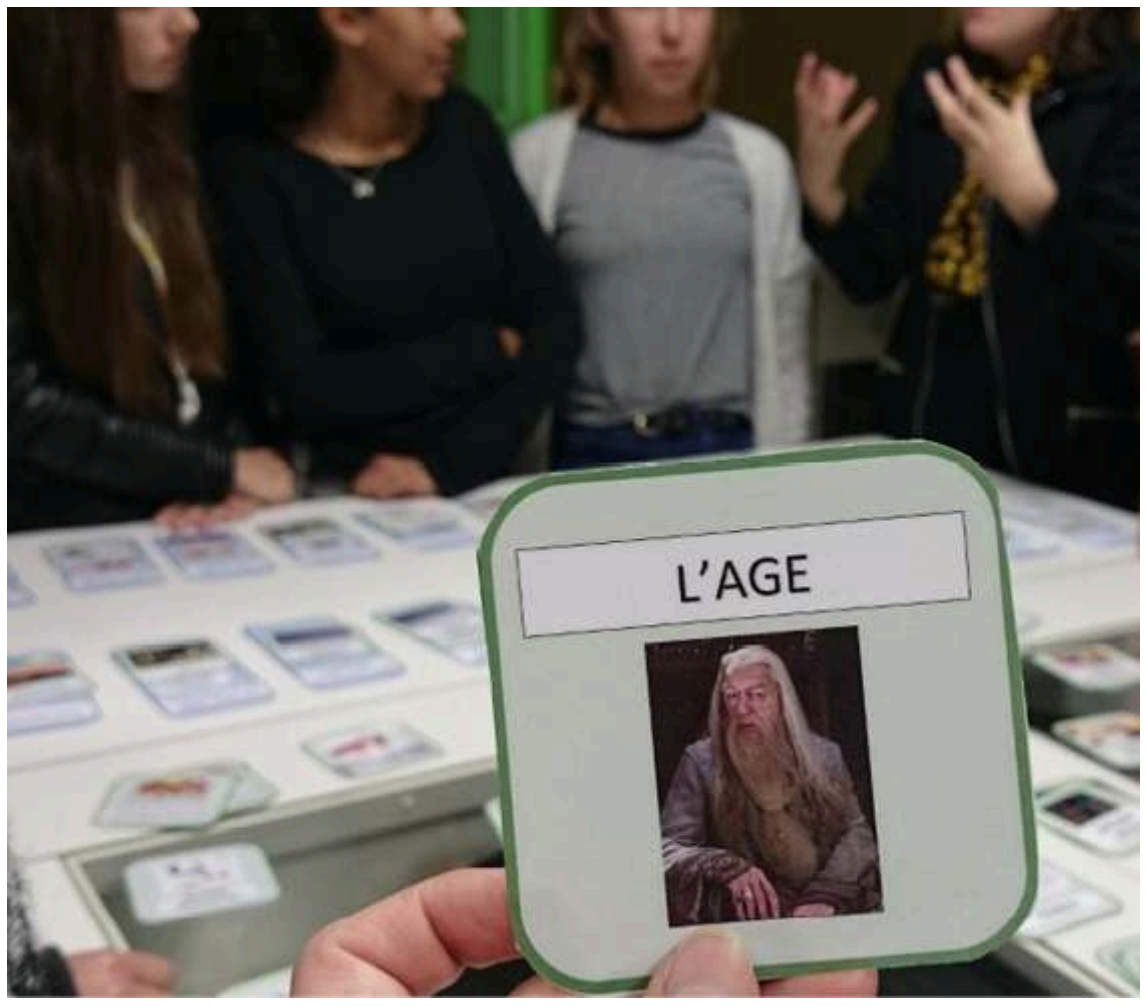

(c) Le Dôme/Virginie Klauser

\section{Didactique et grand public : 3 min pour convaincre}

Le concours Ma thèse en 180 secondes est inspiré de Three minute thesis (3MT' open_in_new), conçu à l'université du Queensland en Australie. Les doctorants se mettent en scène pour présenter leur sujet de recherche, en français et en termes simples. Le tout devant un auditoire profane et diversifié. L'exposé doit être clair, concis et néanmoins convaincant sur son projet de recherche et en trois minutes seulement à l'aide d'une seule diapositive. Le dispositif se déploie dans les pays francophones à partir de 2012 lorsque l'Association francophone pour le savoir (Acfas open_in_new) au Québec décide de l'organiser.

Depuis 2017, le Dôme accompagne cette dynamique en partenariat avec Normandie université et la délégation Normandie du CNRS. Il se charge de proposer une formation adaptée au dispositif. C'est l'occasion, à nouveau, de faire grossir la communauté normande et d'étendre leurs liens.

14 Dans ce cadre, les journées de formation rassemblent une trentaine des doctorants prétendants au concours. Ils sont accompagnés par un minuteur qui rythme les exercices afin d'arriver au format des 3 minutes. Comment synthétiser sa thèse en 3 mots, avec un champ lexical différent du leur en 1 minute? Quelle métaphore utiliser pour être compréhensible par tous? Ils découvrent également des techniques de relaxation car ce format est très intense. S'il génère du stress, il fait aussi monter l'adrénaline et certains deviennent spécialistes pour jouer avec les nerfs du public en 
terminant sur le fil du chrono. Tandis que le rôle des médiateurs est d'accompagner au mieux chacun sur ses points forts - tout en respectant sa personnalité - le challenge pour ces candidats est de prouver qu'ils sont capables de gérer ces contraintes, de pouvoir parler de leur recherche de façon simple, mais pas simpliste, et surtout de passer un bon moment.

\section{Se raconter sur Instagram} manière virtuelle, avec le projet Réflexion.s. Imaginé par Normandie université, le Dôme et le CNRS Normandie, Réflexion.s : instantanés de recherche souhaite mettre en avant les jeunes talents de la recherche régionale en invitant les publics à suivre leur quotidien au travers des médias sociaux. Ils ont l'opportunité de les rencontrer à l'occasion d'évènements de culture scientifique et technique. La médiation passe par Instagram où est présenté le quotidien de jeunes chercheurs normands et Echosciences Normandie ${ }^{4}$ qui diffuse des connaissances. et 10 ont été retenus. Le projet a commencé avec des temps de formation à la médiation scientifique et un accompagnement dans des activités conviviales afin de créer une communauté spécifique. Au travers de l'animation d'un compte Instagram et d'opérations de médiation scientifique (Atelier du chercheur, Ma thèse en 180 secondes, Pint of science...), ces jeunes ambassadeurs ont mis en lumière et partagé leurs travaux de recherche et leurs univers.

\section{Rencontrer son public un verre à la main}

festival Pint of science est l'occasion de sortir la science et les scientifiques des murs $\mathrm{du}$ laboratoire pour les faire s'installer au plus proche des publics, dans les pubs. Imaginé en 2013 en Angleterre par Michael Motskin et Praveen Paul, Pint of science est organisé en France depuis 2014. Le festival se déroule aujourd'hui dans plus de 50 villes et propose chaque année plus de 300 soirées grâce à la mobilisation de 400 bénévoles.

En Normandie, le Dôme accompagne depuis 2019 les doctorants dans sa mise en œuvre sur le terrain, au Havre, à Caen et à Cherbourg-en-Cotentin. Une formation, plébiscitée par les intervenants et les organisateurs, s'est tenue sur Skype pour Le Havre et en direct de Caen. Pour la première fois avec cette manifestation, 16 personnes de deux villes ont bénéficié simultanément de conseils pour adapter leur intervention dans le contexte d'un bar.

19 L'événement a de nombreux avantages : il permet de fédérer, autour des doctorants qui interviennent, des collègues de laboratoire qui viennent en curieux - et souhaitent passer une bonne soirée dans une ambiance conviviale - ou qui collaborent pour enrichir la présentation avec deux points de vue complémentaires. Cela permet également de motiver certains qui, découvrant le format, projettent d'intervenir l'année suivante. C'est l'occasion aussi de faire venir famille et amis qui peuvent « enfin! » comprendre le sujet, vulgarisé pour une présentation courte.

Enfin, parmi le public, se trouvent des personnes qui se déplacent spécialement pour la thématique et profitent de ce moment pour questionner les chercheurs sur des sujets d'actualité. 
Pour Réflexion.s : Instantanés derecherche, des illustrationsminimalistes sont imaginées sur lessujets de thèse des doctorants.
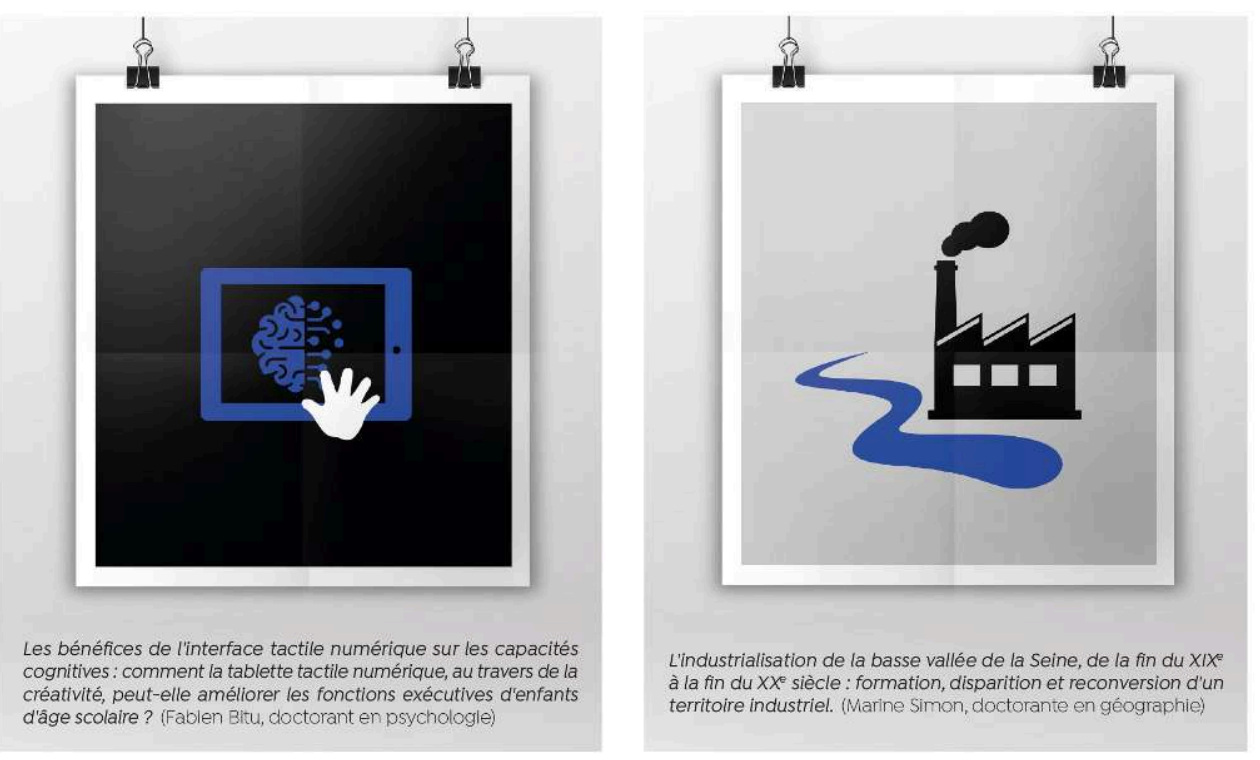

\section{Conclusion}

Le défi que s'est lancé le Dôme est d'assurer la continuité avec ces jeunes chercheurs afin qu'ils poursuivent leur rôle de communicants auprès du public profane. Pour cela, nous cherchons à maintenir le lien avec eux, le plus longtemps possible, grâce à une véritable communauté créée au fil des actions de médiation scientifique. L'objectif est double: les emmener sur les nombreuses opérations à destination des publics, mais aussi, imaginer ensemble de nouveaux formats de médiation.

Pour finir, il s'agit encore de convaincre l'institution de l'utilité de ces formations pour le côté professionnel et de favoriser la reconnaissance de ces compétences à travers, notamment, des open badges.

\section{BIBLIOGRAPHIE}

\section{Echosciences Normandie}

Dossier sur les badges ouverts : www.echosciences-normandie.fr/communautes/le-dome/ dossiers/badges-ouverts-numeriques

Dossier de la communauté sur le quotidien des jeunes chercheurs et chercheuses pour découvrir la recherche « made in Normandie » avec le Projet Réflexion.s : www.echosciences-normandie.fr/ communautes/reflexion-s 


\section{Le Dôme}

Présentation de l'offre doctorale (formation et mise en pratique) du Dôme à destination des doctorants et post-doctorants de la Région Normandie : http://ledome.info/index.php? page=page\&id_manifestation $=1828$

\section{NOTES}

1. La méthode Pecha Kucha est une forme de pitch qui permet de présenter efficacement une idée, un projet, un produit, etc. et ce, dans un laps de temps très limité et clairement défini. Présentation sur Wikipedia : https://fr.wikipedia.org/wiki/Pecha_Kucha

2. Communauté de personnes, souvent auto-organisées, qui partagent de manière collaborative leurs connaissances, pratiques et outils afin d'adapter leurs méthodes, valoriser et faire évoluer leur métier.

3. L'Experimentarium est un programme conçu par l'université de Bourgogne en 2001. Son objectif est d'accompagner les jeunes chercheurs et de créer des rencontres avec différents publics. Depuis 2015, l'initiative s'est élargie en réseau des Experimentarium.

4. Echosciences Normandie se définit comme le réseau social des amateurs de sciences et de technologies du territoire. Il est possible sur la plateforme de s'identifier, poster et découvrir des actualités, entrer en contact avec d'autres membres du réseau, etc.

\section{RÉSUMÉS}

Centre régional de culture scientifique, technique et industrielle à Caen, le Dôme propose différents formats de rencontres entre chercheurs et publics. L'auteur présente un panorama d'actions récentes, comme les open badges, développées par la structure pour accompagner et former les doctorants et post-doctorants de Normandie à la médiation scientifique.

\section{INDEX}

Mots-clés : animation scientifique, chercheur, médiation, centre de sciences, CCSTI

\section{AUTEUR}

\section{VIRGINIE KLAUSER}

Chargée de projet en médiation à destinationdes publics scolaires et doctorants au Dôme

vklauser@ledome.info 